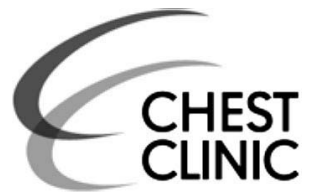

'Department of Medicine, School of Medicine, Chiba University, Chiba, Japan ${ }^{2}$ Department of Respirology, Graduate School of Medicine, Chiba University, Chiba, Japan

\section{Correspondence to}

Kazushi Fujimoto, Department of Medicine, School of Medicine, Chiba University, Chiba 2608670, Japan;

kazfujimoto18@yahoo.co.jp

Received 2 November 2016 Revised 11 December 2016 Accepted 13 December 2016 Published Online First 9 January 2017

\title{
Primary racemose haemangioma of the right bronchial artery with thrombus and slow meandering blood flow
}

\author{
Kazushi Fujimoto, ${ }^{1}$ Hajime Kasai, ${ }^{2}$ Toshihiko Sugiura, ${ }^{2}$ Nobuhiro Tanabe, ${ }^{2}$ \\ Koichiro Tatsumi ${ }^{2}$
}

A 48-year-old man was admitted to our hospital because of an abnormal lung shadow detected on health check-up. He had no history of lung disease. Chest X-ray showed a nodule overlapping the pulmonary artery at the right hilar area; there was no other abnormality (figure 1A). Contrast-enhanced CT of the chest showed hyperplasia of a right racemose haemangioma of a bronchial artery (RHBA), with an area of low density that was suspected as thrombus or malignant tumour (figures 1B, C). Bronchial arteriography showed a pattern that was similar to the beans of a pagoda tree (an herbal medicine) and revealed a blood flow that was disproportionally slow to the artery branch and was meandering in the right RHBA. In addition, there was a relatively small shunt to the pulmonary artery at the right lower lobe (figure 2A). CT scan during bronchial arteriography revealed the detailed structure of the right RHBA (figure 2B). Echocardiography showed no right heart dysfunction, and the tricuspid regurgitation pressure gradient was $15.6 \mathrm{~mm} \mathrm{Hg}$. Malignancy was excluded by positron emission tomographyCT. Therefore, the low-density area was considered a thrombus in the RHBA. We diagnosed primary right RHBA with thrombus. Considering the risk of haemoptysis, we performed coil embolisation of the RHBA. No complication occurred during and after embolisation.

Primary RHBA has been considered a congenital abnormality that is characterised by enlarged and convoluted bronchial arteries with shunt to the

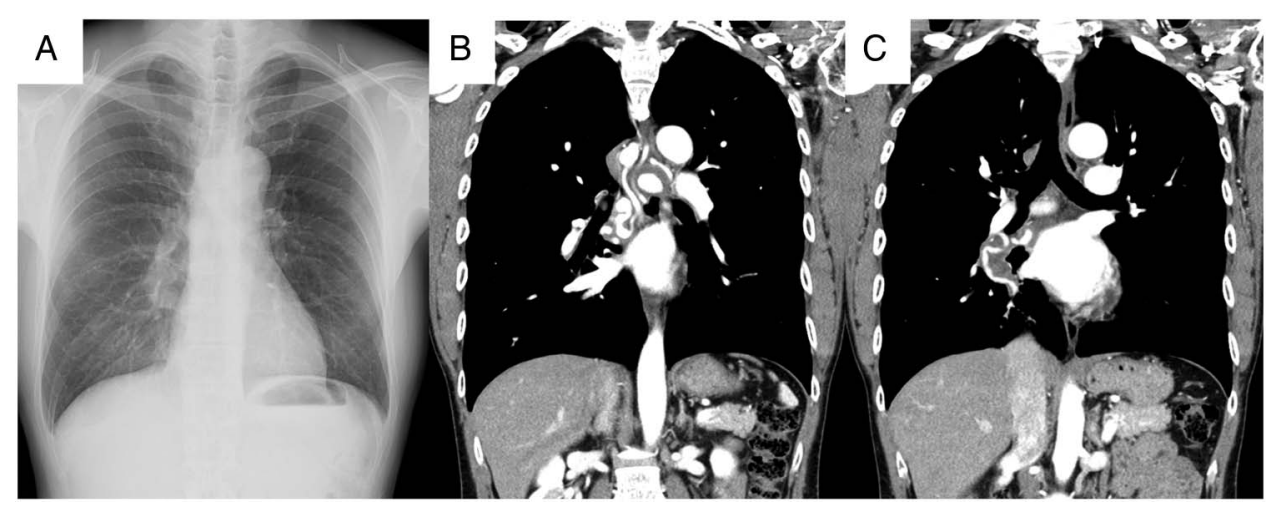

Figure 1 (A) Chest X-ray showing a nodule overlapping the pulmonary artery at the right hilar area. $(B, C)$ Contrastenhanced CT of the chest showing hyperplasia of a right racemose haemangioma of a bronchial artery with an area of low density.

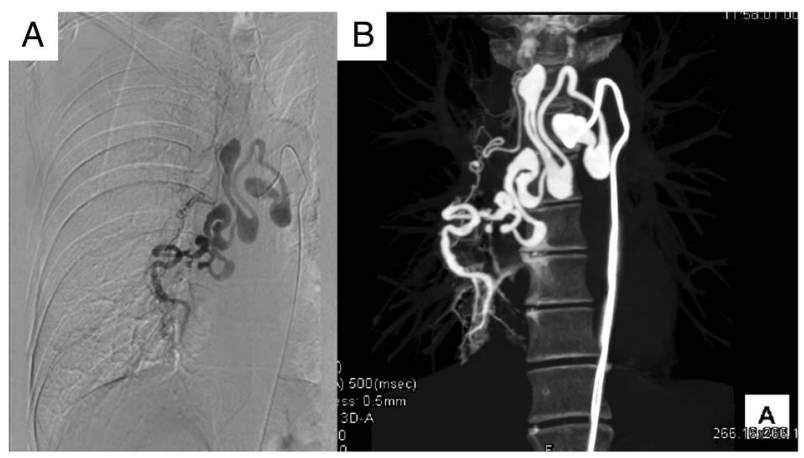

Figure 2 (A) Bronchial arteriography showing a pattern that was similar to the beans of a pagoda tree (an herbal medicine), and a relatively small shunt to the pulmonary artery at the right lower lobe. (B) CT scan during bronchial arteriography showing the detailed structure of the right racemose haemangioma of a bronchial artery. 
pulmonary arteries or veins. ${ }^{1}$ Although primary RHBA may arise from inborn malformation, the genetic risk factor has not been proven. The first case of RHBA was reported by $\mathrm{v}$ Babo et al in $1976 .^{2}$ RHBA has been classified into two types: congenital vascular malformation as a primary form; and subsequent vascular malformation by broncho-pulmonary inflammation as a secondary form. ${ }^{3}$ The prevalence of RHBA is unknown, although cases of RHBA are mainly reported from Japan. ${ }^{4}$ Some reports may describe RHBA as "hypervascularity, dilation and tortuosity of bronchial arteries 5 " and "bronchial artery varices" ${ }^{6 \%}$. Narato et al summarised 34 RHBA cases (16 men, 18 women; mean age 49 years, range $19-80$ years) and demonstrated that $74 \%$ of the patients presented with haemoptysis. Additionally, RHBA often occurred in the right lung (73\%), especially in the right lower lobe $(35 \%) .^{7}$ It is rare to detect RHBA incidentally on routine health check-up. Thrombus is rarely formed in RHBA. In fact, we could not find any reports of RHBA with thrombus.

We present a case of asymptomatic RHBA detected by chest $\mathrm{X}$-ray and considered as primary RHBA without abnormality in the lung fields. For this patient, the possibility of fatal haemoptysis convinced him to undergo coil embolisation.

In conclusion, RHBA should be considered in the differential diagnosis of a chest X-ray finding of nodule overlapping the pulmonary artery at the hilar area. Additionally, RHBA can resemble a pattern of pagoda tree beans on bronchial arteriography and may have thrombus formation.

Contributors $\mathrm{KF}$ is the guarantor of this manuscript and contributed to writing and reviewing of the entire manuscript. HK and TS contributed to image analysis and critical review of the manuscript. NT and KT contributed to critical review of the manuscript.

Competing interests None declared.

Patient consent Obtained.

Provenance and peer review Not commissioned; externally peer reviewed.

\section{REFERENCES}

1 Narita $\mathrm{Y}$, Kojima $\mathrm{H}$, Honjo $\mathrm{S}$, et al. Asymptomatic primary racemose hemangioma of the bronchial artery showing remarkable enlarged and convulted vessels. J Jpn Soc Resp Endoscopy 2009;31:152-8.

$2 \vee$ Babo H, Huzly A, Deninger HK. Angiomas and angioma-like changes of the bronchial arteries (author's transl). Rofo 1976;124:103-10.

3 Cain $\mathrm{H}$, Spanel K. Etiology and morphogenesis of the socalled bronchial arterioma (author's transl). Klin Wochenschr 1980;58:347-57.

4 Shimizu H, Tanabe N, Tatsumi K. [Racemose hemangioma of the bronchial artery]. Nippon Rinsho 2009;67:1038-41.

5 Park GY, Lee KY, Yoo CG, et al. Bronchoscopic findings of endobronchial vascular lesions in patients with haemoptysis. Respirology 1999;4:401-4.

6 Swanson KL, Johnson CM, Prakash UB, et al. Bronchial artery embolization : experience with 54 patients. Chest 2002;121:789-95.

7 Narato $\mathrm{R}$, Enomoto $\mathrm{T}$, Ono $\mathrm{H}$, et al. [A case of successful bronchial artery embolization for primary racemose hemangioma with massive hemoptysis]. Nihon Kokyuki Gakkai Zasshi 2006;44:641-6. 